\title{
Influence of oral health condition on swallowing and oral intake level for patients affected by chronic stroke
}

\author{
Cláudia T Mituuti' \\ Vinicius C Bianco ${ }^{2}$ \\ Cláudia G Bentim ${ }^{3}$ \\ Eduardo $C$ de Andrade' \\ José H Rubo \\ Giédre Berretin-Felix' \\ 'Speech Language and Hearing \\ Department, ${ }^{2}$ Department of \\ Prosthodontics, Bauru School of \\ Dentistry/University of São Paulo, \\ Bauru, Brazil; ${ }^{3}$ SORRI-BAURU, \\ Bauru, Brazil
}

This article was published in the following Dove Press journal:

Clinical Interventions in Aging

16 December 2014

Number of times this article has been viewed

Background: According to the literature, the occurrence of dysphagia is high in cases of stroke, and its severity can be enhanced by loss of teeth and the use of poorly fitting prostheses.

Objective: To verify that the status of oral health influences the level of oral intake and the degree of swallowing dysfunction in elderly patients with stroke in chronic phase.

Methods: Thirty elderly individuals affected by stroke in chronic phase participated. All subjects underwent assessment of their oral condition, with classification from the Functional Oral Intake Scale (FOIS) and nasoendoscopic swallowing assessment to classify the degree of dysphagia. The statistical analysis examined a heterogeneous group $(\mathrm{HG}, \mathrm{n}=30)$ and two groups designated by the affected body part, right (RHG, $n=8$ ) and left (LHG, $n=11$ ), excluding totally dentate or edentulous individuals without rehabilitation with more than one episode of stroke.

Results: There was a negative correlation between the need for replacement prostheses and the FOIS scale for the HG $(P=0.02)$ and RHG $(P=0.01)$. Differences in FOIS between types of prostheses of the upper dental arch in the LHG $(P=0.01)$ and lower dental arch in the RHG $(P=0.04)$. A negative correlation was found between the number of teeth present and the degree of dysfunction in swallowing liquid in the LHG $(P=0.05)$. There were differences in the performance in swallowing solids between individuals without prosthesis and those with partial prosthesis in the inferior dental $\operatorname{arch}(P=0.04)$ for the HG.

Conclusion: The need for replacement prostheses, type of prostheses, and the number of teeth of elderly patients poststroke in chronic phase showed an association with the level of oral intake and the degree of oropharyngeal dysphagia.

Keywords: deglutition, mouth rehabilitation, aged, prosthodontics, dysphagia, cerebrovascular disorders

\section{Introduction}

The process of swallowing physiologically changes with aging. The loss of teeth, very common in this population, is related to the reduction of bone tissue, receptors (proprioceptors and periodontal ligaments), and muscle atrophy. Consequently, orofacial functions are impaired in toothless individuals. ${ }^{1}$ In addition, prostheses are functionally less efficient when compared to individuals with natural dentition. ${ }^{1,2}$

The stability of the jaw occlusion of the posterior teeth or prostheses is important for the swallowing function. ${ }^{2}$ The dental condition and the use of poorly fitting prostheses may contribute to the difficulties arising from old age, and the oral diet and nutritional status of the elderly may be affected by changes related to aging ${ }^{3}$ and changes in skill and desire to feel, bite, chew ${ }^{4}$ and swallow food, ${ }^{3,5}$ significantly compromising and further aggravating the quality of life of these individuals. ${ }^{6}$ 
The literature has shown the influence of oral health condition on swallowing and nutrition, indirectly influencing the daily life activities of the elderly. ${ }^{7}$ Furthermore, it is clear that maintenance of oral health is essential for the general health, quality of life, chewing ability ${ }^{8}$ and, mainly, the reduction of pneumonia risk in fragile elderly people, ${ }^{8,9}$ for it is already known that bacterial colonies in oropharyngeal tissues and in dental plaque are the greatest precursors for the development of aspiration respiratory infections. ${ }^{10}$

Besides the influence of the loss of dentition, the functional impairments resulting from aging may be potentiated by neurological changes resulting in dysphagia in patients, an important cause of morbidity and mortality in this population. ${ }^{11}$

In the adult and elderly population, dysphagia is most commonly associated with stroke. ${ }^{12}$ More than half of patients who have suffered stroke present between six and ten types of disability, muscle weakness being the most prevalent one, followed by communication, speech, and swallowing disorders. ${ }^{13}$ Specifically regarding swallowing, the stroke entails increased oropharyngeal transit time, ${ }^{14,15}$ changes in motor control of tongue, ${ }^{16}$ as well as the presence of laryngotracheal aspiration of food. ${ }^{16,17}$

Once the physiological changes resulting from aging in swallowing may be aggravated by loss of teeth and the use of poorly fitting prostheses, the severity of dysphagia could be influenced by the oral health condition and use of dental prostheses in elderly patients with neurological diseases. However, according to the literature studied, no research has made the correlation between the findings of the swallowing performance with oral health in elderly patients after stroke. Therefore, the aim of this study was to relate the condition of oral health to the level of oral intake and the degree of swallowing dysfunction in elderly patients with stroke in chronic phase.

\section{Methods}

\section{Study design and subjects}

Thirty poststroke individuals, aged 61 to 90 years, whose injury happened 6 months to 9 years previously to their participation in this prospective study. Table 1 shows the characterization data of the sample.

The inclusion criteria considered the following cases: affected by stroke as attested by a medical report, through clinical diagnosis and imaging, with a minimum time of 6 months; aged over 60 years, being in regular clinical neurological monitoring, not having undergone dysphagia rehabilitation; presenting general stable health that would enable the realization of the proposed tests.
Table I Characterization data of the sample of elderly individuals affected by stroke

\begin{tabular}{ll}
\hline Age $\bar{X}$ years (SD) & $73.03(8.59)$ \\
Sex & \\
$\quad$ Female, $n(\%)$ & $15(50)$ \\
$\quad$ Male, $n(\%)$ & $15(50)$ \\
Compromised hemibody & \\
$\quad$ Right, $\mathrm{n}(\%)$ & $10(33.33)$ \\
Left, $\mathrm{n}(\%)$ & $20(66.67)$ \\
Time since injury, $\overline{\mathrm{X}}$ months (SD) & $37.67(27.14)$ \\
Number of episodes of stroke & \\
I, n (\%) & $21(70)$ \\
More than I, n (\%) & $9(30)$ \\
\hline
\end{tabular}

Abbreviations: $\mathrm{n}$, number; SD, standard deviation.

In order to achieve a greater delineation of the group, in addition to considering the group of 30 individuals (heterogeneous group $[\mathrm{HG}])$, the total dentate, the total edentulous without dental prostheses, and individuals with more than one episode of stroke were excluded and the participants were divided into two groups, according to the affected body side: right hemiplegic group (RHG, $\mathrm{n}=8$ ) and left hemiplegic group (LHG, $\mathrm{n}=11$ ). A distribution and recruitment fluxogram is shown in Figure 1.

\section{Procedures}

\section{Oral condition evaluation}

The participants underwent assessment of various components of oral health through traditional health indicators, based on the presence or absence of disease. The data were collected from the clinical examination by a dentist as instructed by the WHO - Oral Health Surveys: Basic

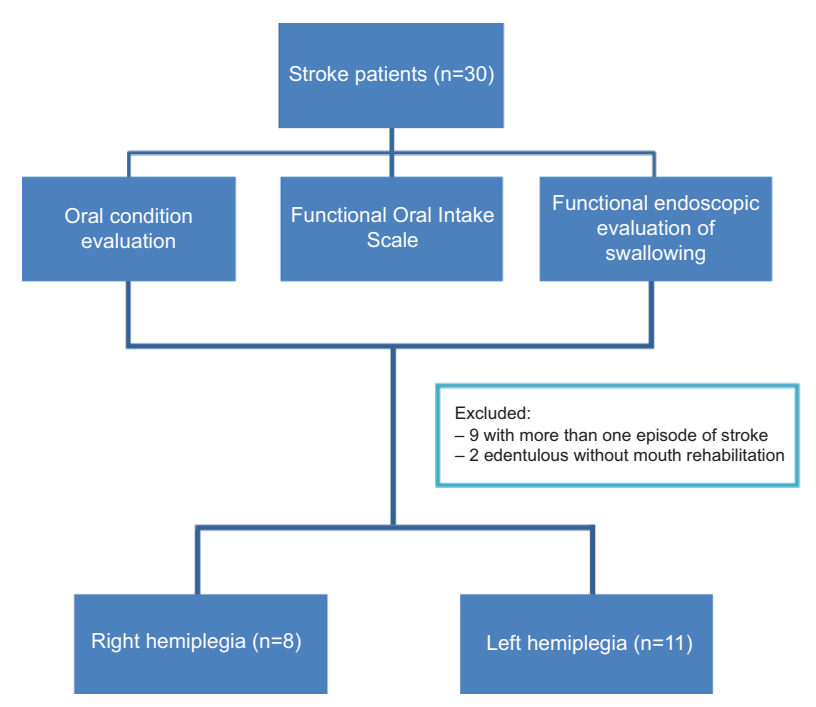

Figure I Recruitment and distribution flowchart. 
Methods, ${ }^{18}$ including the assessment of dental status by the number of decayed, missing, and filled teeth (DMFT) and evaluation of dental prostheses.

The prostheses were evaluated for retention, functional stability, smile aesthetics, degree of bone resorption, and quality of the mucosa. ${ }^{19}$

From the evaluation, the dental arches were classified as without prosthesis or with the presence of partial or complete dental prosthesis; later, the use or need for replacement of dental prostheses was indicated. The need for prostheses replacement was as follows: 0 , no need to change; 1 , partial dental prosthesis was necessary in one of the dental arches; 2 , need for partial dental prosthesis in both dental arches; 3 , need for complete dental prosthesis in one of the dental arches; 4, need for dental prosthesis in both dental arches.

\section{Functional Oral Intake Scale}

Research of oral ingestion was performed by reviewing the usual food consumption patterns referred to in the 24-hour dietary recall. From the data obtained by the research of oral ingestion, patients were classified according to the levels of the Functional Oral Intake Scale (FOIS), ${ }^{20}$ on a scale from I to VII, considering the diet characteristics based on the properties and texture of the food. ${ }^{21}$

\section{Fiberoptic endoscopic evaluation of swallowing}

The fiberoptic endoscopic evaluation of swallowing (FEES) was performed by an otorhinolaryngologist physician collaboratively with the speech therapist. Subjects were asked to remain seated with their heads arranged in the direction of the body axis, without bending or rotation, and the examination was performed using a flexible endoscopic equipment of fiberoptic bronchoscopic type (model Olympus CLV-U20) and an Olympus OTV-SC nasopharyngoscope (Olympus Corporation, Tokyo, Japan).

Three consistent standardized foods were evaluated: liquid (10 mL of filtered water), thick pudding $(10 \mathrm{~mL}$ of thickened dietary grape juice, reaching a final consistency similar to that of pudding), and solid (half a slice of bread, $1 \mathrm{~cm}$ thick). From the data obtained by the FEES, the severity rating of the swallowing disorder was determined in accordance with the scale of functional impairment of swallowing of Macedo Filho, ${ }^{22,23}$ which subdivides the dysphagia severity levels (normal, mild, moderate, and severe).

\section{Statistical analysis}

Spearman's test was used to verify the correlation between the oral health condition (DMFT, number of teeth present, and the need for prostheses replacement) and the performance in the swallowing function (classification in the FOIS scale and dysphagia rating in each consistency). For this analysis to be performed, the FOIS scale and the degree of dysphagia were numerically classified from 1 to 7 and from 1 to 4 , respectively.

For comparison of FOIS results and dysphagia classification, among the different types of prostheses used in the upper and lower dental arches (complete dental prosthesis, partial dental prosthesis, denture or none), the Kruskal-Wallis test was used, followed by the Dunn's test.

\section{Results}

According to the evaluation of oral condition, the mean of DMFT of elderly individuals affected by stroke in this study was 28.7, while the mean of remaining teeth was 5.6, showing that most individuals were edentulous. Table 2 shows the types of prostheses used by the elderly individuals affected by stroke in each dental arch and the need for prostheses replacement.

According to the results of the functional oral intake scale, no individuals were found to be on a single consistency diet or tube dependent. Most individuals (53\%) presented as level V on the FOIS, followed by level IV (34\%) and level VII (13\%).

With the severity rating of the swallowing disorder, no occurrence of severe dysphagia was verified. The distribution of the elderly affected by stroke according to severity rating of the swallowing disorder is shown in Table 3, as well.

Table 2 Distribution of elderly individuals affected by stroke, according to dental prostheses in each arch and the need of prostheses replacement

\begin{tabular}{ll}
\hline $\begin{array}{l}\text { Dental prostheses } \\
\text { Upper, } \mathrm{n}(\%)\end{array}$ & $2(7)$ \\
$\quad$ Dentate & $21(70)$ \\
$\quad$ Complete prostheses & $2(7)$ \\
Partial prostheses & $5(17)$ \\
$\quad$ Without prostheses & \\
Lower, $\mathrm{n}(\%)$ & $0(0)$ \\
$\quad$ Dentate & $10(33)$ \\
$\quad$ Complete prostheses & $4(13)$ \\
$\quad$ Partial prostheses & $16(53)$ \\
$\quad$ Without prostheses & \\
Need for replacement prosthesis, $\mathrm{n}(\%)$ & $9(30)$ \\
No need to change & $4(13)$ \\
Partial dental prosthesis in one dental arch & $3(10)$ \\
Partial dental prosthesis in both dental arches & $5(17)$ \\
Complete dental prosthesis in one dental arch & $9(30)$ \\
Complete dental prosthesis in both dental arches & \\
\hline
\end{tabular}

Abbreviation: $\mathrm{n}$, number. 
Table 3 Distribution of elderly individuals affected by stroke according to classification of severity rating of the swallowing disorder

\begin{tabular}{|c|c|c|c|}
\hline \multicolumn{4}{|c|}{ Severity rating of swallowing disorder } \\
\hline & \multicolumn{3}{|c|}{ Stroke $(n=30)$} \\
\hline & $\begin{array}{l}\text { Normal } \\
\text { n (\%) }\end{array}$ & $\begin{array}{l}\text { Mild } \\
\text { n (\%) }\end{array}$ & $\begin{array}{l}\text { Moderate } \\
\text { n (\%) }\end{array}$ \\
\hline Liquid & $5(17)$ & $9(30)$ & $16(53)$ \\
\hline Pudding & $14(47)$ & $5(17)$ & II (37) \\
\hline Solid & $13(43)$ & $6(20)$ & II (37) \\
\hline
\end{tabular}

Abbreviation: $\mathrm{n}$, number.

According to the results of the statistical analysis, correlations between the oral health condition and the results of the FOIS scale in elderly individuals affected by stroke in this study were found. There was a statistically significant negative correlation between the need for replacement of prostheses and the rating scale, and also in the HG and in RHG, showing that the greater the need for replacement of the prosthesis, the worse the rating of the FOIS scale.

There were differences in the classification of the FOIS scale, for the LHG, in the different types of prostheses used in the upper dental arch, whereas for the RHG the difference in the classification of FOIS occurred between groups of individuals with different types of prostheses used in the lower dental arch. After completion of the Dunn's post-test, the difference was confirmed only in the LHG, demonstrating that individuals of this group with partial prostheses in the upper arch (ie, that still had natural elements and whose missed teeth had been rehabilitated) had better ratings in the FOIS scale compared to individuals with total prostheses.

The results of the correlation between the FOIS scale and the oral health condition are shown in Table 4.
In the statistical analysis between the degree of swallowing dysfunction and oral health condition, a significant negative correlation between the number of teeth and the swallowing performance of liquid in the LHG was found, demonstrating that the greater the number of teeth, the lower the degree of swallowing dysfunction.

According to the Kruskal-Wallis test, there was no difference in the performance of swallowing solid boluses depending on the type of prosthesis used in the lower dental arch; in the HG, however, when applying the post-Dunn's test, this difference was not confirmed $(P>0.05)$. The comparison of the FOIS scale between the types of dental prostheses is shown in Table 5.

The results of the correlation between the degree of dysphagia and the oral health condition are shown in Table 6, and the comparison of the degree of swallowing dysfunction between types of dental prostheses is described in Table 7.

\section{Discussion}

According to the literature, individuals affected by stroke often exhibit decreased level of consciousness, paralysis of the muscles involved in swallowing, sensory deficits of the pharynx and oral cavity, as well as loss of appetite. ${ }^{24} \mathrm{With}$ these disorders related to swallowing, dysphagia becomes an important symptom in this population, and may be aggravated by teeth loss and the use of poorly fitting prosthesis, common during aging. Furthermore, the hypothesis that oropharyngeal colonization is primarily responsible for the subsequent respiratory infection, owing to aspiration, is strongly supported by the literature. ${ }^{10}$

No literature studies have made the correlation between the findings of the swallowing performance and the condition of the oral health in elderly patients after stroke.

Table 4 Spearman correlation coefficient and $P$-values obtained in the correlation between the results of the oral condition and the level of oral intake, ranked according to the FOIS scale of 30 elderly individuals affected by stroke (results grouped according to hemiplegia)

\section{FOIS}

\begin{tabular}{|c|c|c|c|c|c|c|}
\hline & \multirow{2}{*}{\multicolumn{2}{|c|}{$\begin{array}{l}\text { HG } \\
(n=30)\end{array}$}} & \multicolumn{4}{|l|}{ I episode } \\
\hline & & & \multicolumn{2}{|l|}{ RHG $(n=8)$} & \multicolumn{2}{|l|}{ LHG $(n=I I)$} \\
\hline & Correlation & $P$ & Correlation & $P$ & Correlation & $P$ \\
\hline DMFT & -0.02 & 0.92 & 0.43 & 0.27 & -0.23 & 0.48 \\
\hline Teeth present & 0.11 & 0.55 & -0.43 & 0.27 & $0.4 \mathrm{I}$ & 0.20 \\
\hline Need to change & -0.42 & $0.02 *$ & -0.86 & $0.01 *$ & -0.03 & 0.92 \\
\hline
\end{tabular}

Note: $* p \leq 0.05$.

Abbreviations: DMFT, number of decayed, missing, and filled teeth; FOIS, Functional Oral Intake Scale; HG, heterogeneous group; LHG, left hemiplegic group; n, number; RHG, right hemiplegic group. 
Table 5 Comparison of the level of oral ingestion between types of dental prostheses, ranked according to the FOIS scale of 30 elderly individuals affected by stroke (results grouped according to hemiplegia)

\begin{tabular}{|c|c|c|c|}
\hline \\
\hline \multirow{2}{*}{ FOIS } & $\begin{array}{l}\text { HG } \\
(n=30)\end{array}$ & $\begin{array}{l}\text { RHG } \\
(n=8)\end{array}$ & $\begin{array}{l}\text { LHG } \\
(n=I I)\end{array}$ \\
\hline & $P$ & $P$ & $P$ \\
\hline Type of dental prosthesis (upper arch) & 0.17 & 0.45 & $0.01 *$ \\
\hline Type of dental prosthesis (lower arch) & 0.54 & $0.04^{*}$ & 0.12 \\
\hline
\end{tabular}

Note: $* P \leq 0.05$

Abbreviations: FOIS, Functional Oral Intake Scale; HG, heterogeneous group; LHG, left hemiplegic group; $n$, number; RHG, right hemiplegic group.

The results of this study showed some correlations between the oral condition and the data of the swallowing performance even when elderly individuals affected by stroke were part of the heterogeneous group.

Besides the statistical analysis for the study group, in an attempt to reduce its heterogeneity, the subjects were divided according to the side of the motor impairment after stroke. Former studies have suggested that dysphagia maybe associated with diffuse lesions in one or both hemispheres, anterior fossa, or brainstem, as swallowing has a bilateral cortical representation. It has been well established that the protection of the airways during swallowing requires a precise coordination with accurate movements of the structures involved and adequate propulsion of the bolus to the oropharynx and esophagus, ${ }^{25}$ thus, the motor ability for swallowing seems to be essential for this protection.

In the HG and RHG, it was found that the higher the need for replacement of the prosthesis, the worse the rating in the
FOIS scale; ie, individuals with greater need for replacement of the prostheses had a greater perception in making more compensation and changes in their diet, which reflected the classification level of oral intake.

The FOIS scale was developed to describe the functional level of the daily oral intake of food and liquid of a patient, considering the changes in diet and need for compensation during swallowing. ${ }^{20}$ These limitations and changes in oral intake result from patient perception and self-perception of swallowing ability that is impaired. Therefore, from the results, it can be inferred that when there was a greater need for replacement of the prosthesis, the patient's perception of doing more compensation and dietary modifications is reflected in the classification of the FOIS scale.

Significant changes in oral condition can have a major effect on the functions of eating and drinking and on the nutritional status of elderly individuals and those with neurological diseases. ${ }^{26}$ Thus, the selection of food is determined by the difficulty in chewing and swallowing of the individuals. Missing teeth and the use of prosthesis cause changes in the chewing ability of the elderly according to the type of food, and influences the swallowing function; ${ }^{27}$ thus, soft or mashed foods are preferentially ingested by the elderly, due to the difficulty in mastication, ${ }^{28}$ showing that there is an influence of oral health condition on the selection of foods.

Besides the influence of the oral condition on swallowing, there was a difference between the types of prostheses and classification of the FOIS scale when the results of the evaluation of individuals with impaired right and left hemiplegia were analyzed separately. The difference between the types of prostheses could be confirmed by post-Dunn's test in the LHG, and demonstrated that individuals with partial dentures

Table 6 Spearman correlation coefficient and P-values obtained in the correlation between the results of the oral condition and the degree of dysphagia of 30 elderly individuals affected by stroke (results grouped according to hemiplegia)

\begin{tabular}{|c|c|c|c|c|c|c|c|}
\hline \multicolumn{8}{|c|}{ Degree of swallowing dysfunction } \\
\hline & & \multicolumn{2}{|l|}{ HG } & \multicolumn{4}{|l|}{ I episode } \\
\hline & & \multicolumn{2}{|l|}{$(n=30)$} & \multicolumn{2}{|l|}{ RHG $(n=8)$} & \multicolumn{2}{|l|}{ LHG $(n=I I)$} \\
\hline & & Correlation & $P$ & Correlation & $\mathbf{P}$ & Correlation & $P$ \\
\hline \multirow[t]{3}{*}{ DMFT } & Liquid & 0.10 & 0.59 & 0.07 & 0.84 & 0.42 & 0.19 \\
\hline & Pudding & -0.11 & 0.95 & -0.45 & 0.24 & 0.30 & 0.37 \\
\hline & Solid & 0.18 & 0.35 & -0.01 & 0.98 & 0.33 & 0.31 \\
\hline \multirow[t]{3}{*}{ Teeth present } & Liquid & -0.17 & 0.38 & -0.07 & 0.84 & -0.59 & $0.05^{*}$ \\
\hline & Pudding & -0.08 & 0.65 & 0.45 & 0.24 & -0.49 & 0.12 \\
\hline & Solid & -0.19 & 0.34 & 0.01 & 0.98 & 0.41 & 0.20 \\
\hline \multirow[t]{3}{*}{ Need to change } & Liquid & -0.15 & 0.44 & 0.06 & 0.88 & 0.21 & 0.52 \\
\hline & Pudding & 0.16 & 0.39 & 0.16 & 0.70 & 0.28 & 0.40 \\
\hline & Solid & 0.04 & 0.83 & 0.42 & 0.30 & 0.14 & 0.67 \\
\hline
\end{tabular}

Note: $* P \leq 0.05$.

Abbreviations: DMFT, number of decayed, missing, and filled teeth; HG, heterogeneous group; LHG, left hemiplegic group; n, number; RHG, right hemiplegic group. 
Table 7 Comparison of the degree of swallowing dysfunction between types of dental prostheses of 30 elderly individuals affected by stroke (results grouped according to hemiplegia)

\begin{tabular}{lllll}
\hline Degree of swallowing dysfunction & & & \\
\hline & & $\begin{array}{l}\text { HG } \\
(\mathbf{n}=\mathbf{3 0})\end{array}$ & $\begin{array}{l}\text { RHG } \\
\mathbf{( n = 8 )}\end{array}$ & $\begin{array}{l}\text { LHG } \\
\mathbf{( n = 1 1 )}\end{array}$ \\
\cline { 3 - 4 } & & $\mathbf{P}$ & $\mathbf{P}$ & $\mathbf{P}$ \\
\hline Type of dental prosthesis & Liquid & 0.07 & 0.44 & 0.11 \\
(upper arch) & Pudding & 0.27 & 0.16 & 0.41 \\
Type of dental prosthesis & Solid & 0.07 & 0.34 & 0.23 \\
(lower arch) & Liquid & 0.16 & 0.68 & 0.62 \\
& Pudding & 0.59 & 0.31 & 0.43 \\
& Solid & $0.04^{*}$ & 0.48 & 0.30 \\
\hline
\end{tabular}

Note: $* P \leq 0.05$.

Abbreviations: HG, heterogeneous group; LHG, left hemiplegic group; n, number; RHG, right hemiplegic group.

had better ratings in the FOIS scale than individuals with complete prostheses. No studies were found relating the oral condition and the rating scale. Thus, we can infer that when there was partial prosthesis rehabilitation along with the presence of some dental elements, subjects needed less compensation and changes in the oral diet. In this sense, studies show that the use of prostheses in the edentulous contributes to the maintenance of the physiological process of swallowing in the elderly, ${ }^{29}$ the fixation of the prosthesis in the mandible, ${ }^{30}$ and the presence of natural elements enables greater preservation of functional swallowing.

When comparing the swallowing performance according to the type of prosthesis used, there was no difference between the types of prostheses fitted in the lower dental arch in swallowing solids in the HG. Studies in healthy elderly subjects demonstrate the importance of the oral condition in the performance of the oral phase of swallowing. Changes in masticatory function related to the oral condition, such as difficulties biting and chewing food ${ }^{27}$ demonstrated an influence on the pharyngeal phase of swallowing, such as premature escape of food, waste retention in the vallecula and pyriform sinuses, and the presence of coughing and gagging. ${ }^{27}$

Moreover, the absence of dental prosthesis can result in a longer duration of swallowing, ${ }^{29}$ and the chewing and swallowing difficulties in healthy older adults decreased after the replacement of removable total prostheses by mandibular implant-supported prostheses..$^{30}$ The literature has shown positive effects in the swallowing function with the use of prostheses and the differences in swallowing performance with prostheses in the upper or inferior arches. ${ }^{8}$ A study with healthy elderly individuals showed the differences between the total duration of swallowing, the latency period before pharynx elevation, duration of preparatory and oral phase, when using or not using the dental prostheses. ${ }^{29}$
Finally, in the LHG, it was observed that the smaller the number of teeth present, the worse the rating of dysphagia for liquid. Although this study aimed at finding an influence of the presence and number of teeth on chewing and consequently on swallowing solids, ${ }^{1,27,28}$ it also demonstrates the influence of the oral condition on liquid swallowing for this population. According to Tamura et al, ${ }^{2}$ especially for liquid food, which demands more coordination during swallowing, stability in the jaw is also required, so that there is greater stability of the hyolaryngeal complex. To start the pharyngeal phase, the rise of the hyoid bone by the suprahyoid muscles and laryngeal elevation should occur. To complement these events, the jaw must be stabilized by natural or rehabilitated teeth into the proper position, because when the elevation of the hyoid bone and larynx is insufficient, there is an increased risk of aspiration during swallowing.

\section{Conclusion}

The findings of this study indicate that the oral health condition of elderly individuals after stroke in chronic phase showed an association with the level of oral intake and the degree of oropharyngeal dysphagia. The limitations of the study should be considered, one being the lack of access to the imaging examinations to characterize the individuals according to the type of stroke, damaged areas, and extent of injury, and heterogeneity of the time of brain involvement, in addition to the effects of medications and other comorbidities, which were not taken into account. Also, owing to the small sample, strong correlations could not be observed, and a cause/effect relationship cannot be determined.

Despite the limitations of this study, and the fact that hemiplegia does not necessarily represent a direct relationship with the injured brain hemisphere contralaterally, since cerebellum lesions may cause ipsilateral motor damages, ${ }^{31}$ the findings indicated that the level of oral intake was influenced by the condition or type of dental prostheses, regardless of the side of the body affected. On the other hand, the number of teeth were demonstrated to be related to the level of dysphagia in individuals with right hemiplegia, which shows that the oral health condition differentially affects the physiological responses of swallowing, influenced by the brain damage.

Considering the present sample, further research is necessary pertaining to the oral condition and the neurological involvement of individuals. In addition, these issues demonstrate the need for an interdisciplinary research team comprising dentists, physicians, speech pathologists, and other health professionals, so as to study and define the treatment and guidelines to patients and caretakers, in order to maintain 
the best oral health condition in the elderly presented with oropharyngeal dysphagia.

\section{Acknowledgments}

This study was approved by the Ethics Committee for Research of FOB/USP under case number 050/2009 and was carried out with the express agreement of the enrolled individuals. We thank the Foundation for Research Support (FAPESP) for the funding for the study.

\section{Disclosure}

The authors declare no conflicts of interest in this work.

\section{References}

1. Müller F, Schimmel M. Tooth loss and dental prostheses in the oldest old. European Geriatric Medicine. 2010;1(4):239-243.

2. Tamura F, Mizukami M, Ayano R, Mukai Y. Analysis of feeding function and jaw stability in bedridden elderly. Dysphagia. 2002; 17(3):235-241.

3. Sura L, Madhavan A, Carnaby G, Crary MA. Dysphagia in the elderly: management and nutritional considerations. Clin Interv Aging. 2012;7:287-298

4. de Oliveira TR, Frigerio ML. Association between nutrition and the prosthetic condition in edentulous elderly. Gerodontology. 2004;21(4):205-208.

5. Palmer CA. Gerodontic nutrition and dietary counseling for prosthodontic patients. Dent Clin North Am. 2003;47(2):355-371.

6. Berretin-Felix G, Nary Filho H, Padovani CR, Trindade Junior AS, Machado WM. Electromyographic evaluation of mastication and swallowing in elderly individuals with mandibular fixed implant-supported prostheses. J Appl Oral Sci. 2008;16(2):116-121.

7. Furuta M, Komiya-Nonaka M, Akifusa S, et al. Interrelationship of oral health status, swallowing function, nutritional status, and cognitive ability with activities of daily living in Japanese elderly people receiving home care services due to physical disabilities. Community Dent Oral Epidemiol. 2013;41(2):173-181.

8. Furuta M, Yamashita Y. Oral Health and Swallowing Problems. Curr Phys Med Rehabil Rep. 2013;1:216-222.

9. Logemann JA, Curro FA, Pauloski B, Gensler G. Aging effects on oropharyngeal swallow and the role of dental care in oropharyngeal dysphagia. Oral Dis. 2013;19(8):733-737.

10. Garcia R. A review of the possible role of oral and dental colonization on the occurrence of health care-associated pneumonia: underappreciated risk and a call for interventions. Am J Infect Control. 2005; 33(9):527-541.

11. Kumar S. Swallowing and dysphagia in neurological disorders. Rev Neurol Dis. 2010;7(1):19-27.

12. Perry L, Love CP. Screening for dysphagia and aspiration in acute stroke: a systematic review. Dysphagia. 2001;16(1):7-18.

13. Lawrence ES, Coshall C, Dundas R, et al. Estimates of the prevalence of acute stroke impairments and disability in a multiethnic population. Stroke. 2001;32(6):1279-1284.
14. Daniels SK, Schroeder MF, DeGeorge PC, Corey DM, Foundas AL, Rosenbek JC. Defining and measuring dysphagia following stroke. Am J Speech Lang Pathol. 2009;18(1):74-81.

15. Power ML, Hamdy S, Goulermas JY, Tyrrell PJ, Turnbull I, Thompson DG. Predicting aspiration after hemispheric stroke from timing measures of oropharyngeal bolus flow and laryngeal closure. Dysphagia. 2009;24(3):257-264.

16. Terré R, Mearin F. Resolution of tracheal aspiration after the acute phase of stroke-related oropharyngeal Dysphagia. Am J Gastroenterol. 2009;104(4):923-932.

17. Warnecke T, Teismann I, Meimann W, et al. Assessment of aspiration risk in acute ischaemic stroke - evaluation of the simple swallowing provocation test. J Neurol Neurosurg Psychiatry. 2008;79(3): 312-314.

18. World Health Organization. Oral health surveys: basic methods. Geneva: World Health Organization, 1997.

19. Corsalini M, Rapone B, Grassi FR, Di Venere D. A study on oral rehabilitation in stroke patients: analysis of a group of 33 patients. Gerodontology. 2010;27(3):178-182.

20. Crary MA, Mann GD, Groher ME. Initial psychometric assessment of a functional oral intake scale for dysphagia in stroke patients. Arch Phys Med Rehabil. 2005;86(8):1516-1520.

21. National Dysphagia Diet Task Force. The National Dysphagia Diet: Standardization for Optimal Care. American Dietetic Association. 2002.

22. Macedo Filho ED. Avaliação Videoendoscópica da Deglutição. VED na abordagem da disfagia orofaríngea. In: Jacobi JS, Levy DS, Silva LMC. Disfagia. Avaliação e Tratamento. Rio de Janeiro: Revinter; 2003:332-342. Portuguese.

23. Santoro PP, Furia CL, Forte AP, et al. Otolaryngology and speech therapy evaluation in the assessment of oropharyngeal dysphagia: a combined protocol proposal. Braz J Otorhinolaryngol. 2011;77(2):201-213.

24. Nakajima M, Takada T, Terasaki Y, Nagano K, Naritomi H, Minematsu K. Clinical significance of oral intake in patients with acute stroke. Dysphagia. 2010;25(3):192-197.

25. Bingjie L, Tong Z, Xinting S, Jianmin X, Guijun J. Quantitative videofluoroscopic analysis of penetration-aspiration in post-stroke patients. Neurol India. 2010;58(1):42-47.

26. Ship JA, Duffy V, Jones JA, Langmore S. Geriatric oral health and its impact on eating. J Am Geriatr Soc. 1996;44(4):456-464.

27. Fazito LT, Perim JV, Di Ninno CQMS. Comparação das queixas alimentares de idosos com e sem prótese dentária. Rev CEFAC. 2004;6(2):143-150. Portuguese.

28. Ow RK, Loh T, Neo J, Khoo J. Perceived masticatory function among elderly people. J Oral Rehabil. 1997;24(2):131-137.

29. Furuya J. [Effects of wearing complete dentures on swallowing in the elderly]. Kokubyo Gakkai Zasshi. 1999;66(4):361-369. Japanese.

30. Berretin-Felix G, Machado WM, Genaro KF, Nary Filho H. Effects of mandibular fixed implant-supported prostheses on masticatory and swallowing functions in completely edentulous elderly individuals. Int J Oral Maxillofac Implants. 2009;24(1):110-117.

31. Small SL, Hlustik P, Noll DC, Genovese C, Solodkin A. Cerebellar hemispheric activation ipsilateral to the paretic hand correlates with functional recovery after stroke. Brain. 2002;125(Pt 7):1544-1547.

Clinical Interventions in Aging

\section{Publish your work in this journal}

Clinical Interventions in Aging is an international, peer-reviewed journal focusing on evidence-based reports on the value or lack thereof of treatments intended to prevent or delay the onset of maladaptive correlates of aging in human beings. This journal is indexed on PubMed Central, MedLine,

CAS, Scopus and the Elsevier Bibliographic databases. The manuscript management system is completely online and includes a very quick and fair peer-review system, which is all easy to use. Visit http://www.dovepress. com/testimonials.php to read real quotes from published authors. 